\title{
Gender Dysphoria and Autism - An Overview
}

\author{
C. Campos ${ }^{1}$, S. Carneiro ${ }^{1}$, B. Jorge ${ }^{1}$, M. Antunes ${ }^{2}$, A.T. Pereira ${ }^{3}$, J. Mesquita
}

${ }^{1}$ Hospital de Braga, Department of Psychiatry and Mental Health, Braga, Portugal.

${ }^{2}$ Centro Hospitalar do Porto, Department of Child and Adolescent Psychiatry, Porto, Portugal.

${ }^{3}$ Centro Hospitalar Vila Nova de Gaia/Espinho, Department of Psychiatry and Mental Health, Vila Nova de Gaia, Portugal.

Background: In clinical services there has been a higher than expected diagnosis of Autism Spectrum Disorders (ASD) among individuals with Gender Dysphoria (GD): while in the general population it is estimated that the diagnosis of ASD occurs in about $1 \%$, researchers investigating rates of $A S D$ in trans people attending services have found that up to $7.8 \%$ report a lifetime prevalence of ASD. It has been of great clinical interest because it has implications for diagnosis and treatment.

Objectives, Materials and Methods: The aim of this work is to explore the relationship between ASD and GD, investigate the developed theories that justify the surprisingly high co-occurrence between them, and to analyze the inherent clinical implications and difficulties. We performed a non-systematic review of the literature of interest on the topic based on a Pubmed search using the terms "Autism Spectrum Disorders" and "Gender Dysphoria".

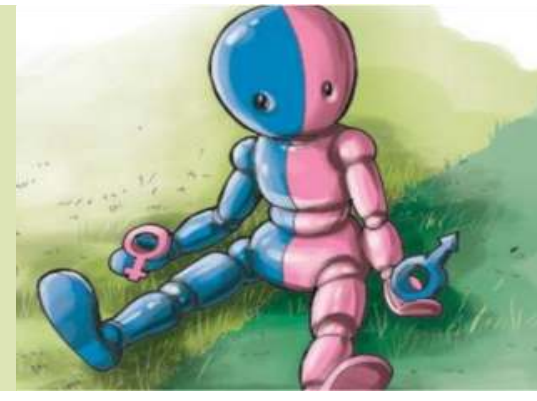

\section{There are several potential explanations for the co-occurrence between GD and ASD:}

Extreme Male Brain theory: Within the general population, typical women are thought to display more empathizing and less systemizing traits compared with typical men. Studies assessing these brain domains in people with ASD have found an extreme version of the male brain, where systemizing is above average and empathy is decreased. It has been hypothesized that high levels of fetal testosterone are responsible for the extreme male brain. This theory could account for the co-occurrence of gender dysphoria and ASD in transmen (natal women), but not in transwomen.

Sexual Hormones theory: Autistic traits displayed during childhood have been positively related to fetal testosterone. Similarly, it is pointed out that one of the possible mechanisms responsible for gender identity may be related to the direct effect of testosterone on the development of the human brain. Thus, increased exposure to male sex hormones represents a common pathway for the development of GD and ASD.

Atypical Development of Gender Identity: The formation of a gender identity depends on cognitive, social and communication skills that are often impaired in individuals with ASD, being that the gender is one of the most intensely socialized constructs, with rules that are often implicit and intuitive, and therefore many times difficult to grasp for an individual with ASD. This impairment in social interaction could contribute to an atypical gender identity formation.

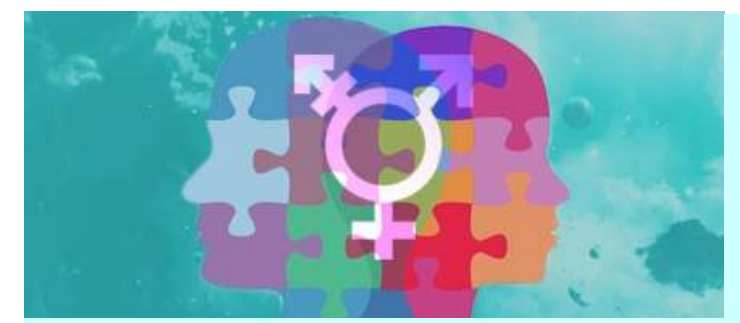

The co-occurrence between ASD and DG is likely to be complex and may depend on several underlying

mechanisms. Many theories for this co-occurrence have been suggested, but almost all are speculative and lack evidence. It can, however, derive from a purely spurious relationship as a result of the characteristics of ASD (for ex. a child having restricted interests centered in activities more typical of the opposite gender), which may confound the diagnosis.
The evaluation of GD in individuals with ASD can be quite complex owing to some of the difficulties experienced by them:

Difficulties in communication, shared psychological conceptualization, and in the building of a therapeutic relationship between the patient and the clinician;

Difficulties in communication and sharing internal experiences that depend on concepts such as the "gender" that is a construct that results from the relationship of the individual with society, which is often compromised in the ASD;

The social role transition that is usually recommended before starting the hormonal or surgical treatment may be difficult for the person to apply, and for the physician to evaluate in this population.

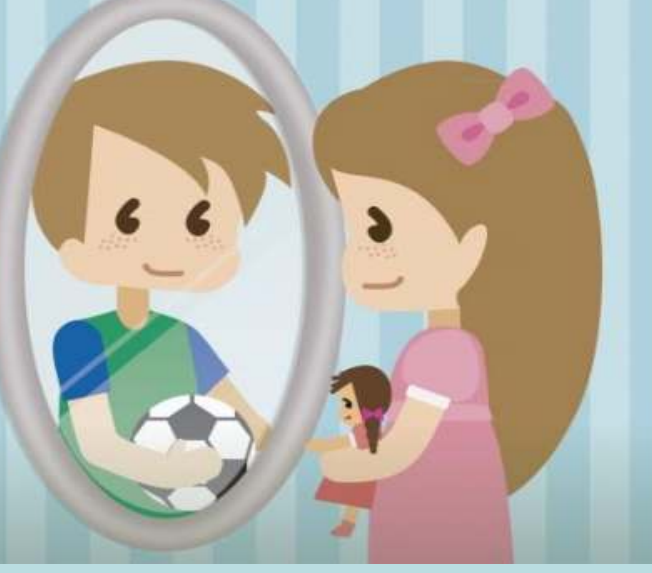

Despite these and other obstacles, individuals with ASD have the same rights as other individuals to appropriate assessment, diagnosis, and treatment of gender-related concerns.

As such, diagnosis and access to therapy should not be prohibited in these individuals, however, the diagnostic phase should be individualized and extended because of the difficulty of differentiation between both conditions.

\section{On suspicion of GD (+/- ASD) we suggest:}

Referral to endocrinology / genetics in order to investigate the potential presence of sexual ambiguity;

- Assessment of the possibility of the individual being a victim of bullying or discrimination / social aggression, of the potential development of psychopathology (more frequently depression and anxiety), and assessing the suicidal risk;

- Helping the individual to explore his/her gender narrative and identity;

- Psychoeducation directed to the patient and his/her family regarding the diagnosis and the therapeutic possibilities in the sense of relief of the suffering;

- Referral to psychiatry services with professionals trained in clinical sexology. 